\title{
Communication \\ Generation of Switchable Chirp Waveforms in the Photonic Domain with Immunity to Dispersion-Induced Power Fading
}

\author{
Chaoqun Huang, Jun Ou * and Hao Chi
}

check for

updates

Citation: Huang, C.; Ou, J.; Chi, H Generation of Switchable Chirp Waveforms in the Photonic Domain with Immunity to DispersionInduced Power Fading. Photonics 2021, 8, 501. https://doi.org/ $10.3390 /$ photonics 8110501

Received: 18 September 2021 Accepted: 4 November 2021 Published: 8 November 2021

Publisher's Note: MDPI stays neutral with regard to jurisdictional claims in published maps and institutional affiliations.

Copyright: (c) 2021 by the authors. Licensee MDPI, Basel, Switzerland. This article is an open access article distributed under the terms and conditions of the Creative Commons Attribution (CC BY) license (https:// creativecommons.org/licenses/by/ $4.0 /)$.
School of Communication Engineering, Hangzhou Dianzi University, Hangzhou 310018, China; huangcq0102@hdu.edu.cn (C.H.); chihao@hdu.edu.cn (H.C.)

* Correspondence: oujun@hdu.edu.cn

\begin{abstract}
A novel photonic approach to generating switchable multi-format chirp waveforms using a dual-drive dual-parallel Mach-Zehnder modulator (DD-DPMZM) is proposed and experimentally demonstrated. By properly controlling the bias voltage on the DD-DPMZM, different chirp RF waveforms, including the dual-, down-, and up-chirp waveforms, can be obtained when an RF signal and a chirp RF signal are injected into the modulator. A main feature of this approach is that it can eliminate chromatic dispersion-induced RF power fading, which is highly desired in distributed multi-functional radars based on radio over fiber. There is no polarization control and optical filtering in the given scheme, which also improves the stability and feasibility of the approach. An experiment successfully demonstrated the generation and switching of the multi-format chirp waveforms and the capability of immunity to dispersion-induced power fading.
\end{abstract}

Keywords: microwave photonics; switchable multi-format chirp waveforms signal; immunity of power fading

\section{Introduction}

Chirp microwave waveforms have been widely employed in modern radars. Chirp waveforms can be compressed at radar receivers, which solves the tradeoff between the detection range and the range resolution of radars [1,2]. Conventionally, chirp waveforms can be generated through electrical methods. However, the center frequency and bandwidth of the electrically generated signals are limited by the inherent electronic bottleneck in electronic devices, such as surface acoustic wave delay lines and digital-to-analog converters. Compared with electrical methods, generating chirp signals in the optical domain [3] has the advantages of high center frequency and bandwidth, large spectral tuning range and anti-electromagnetic interference.

Up to now, many microwave photonic methods for the generation of chirp microwave waveforms have been reported [4]. Representative methods include frequency-to-time mapping [5-8] and optical heterodyning [9-11]. In order to increase the time-bandwidth product of the generated chirp signals, methods based on frequency-sweeping optoelectronic oscillators [12], frequency and bandwidth multiplication using external modulators [13,14] have been proposed. Single-chirp waveforms have the problem of range-Doppler coupling; therefore, dual-chirp microwave waveforms were proposed to overcome this problem. A dual-chirp waveform consists of two complementary linearly chirped waveforms within the same time duration. In some applications, sending dual-chirp microwave waveforms is a better solution. Some photonic approaches to generating dual-chirp waveforms have been reported in recent years, such as schemes using a dual-parallel Mach-Zehnder modulator (DP-MZM) [15], cascading MZMs [16] or a dual-polarization quadrature phase shift keying modulator (DP-QPSK) [17].

In some applications, such as multifunctional radars, in order to meet different requirements, switchable multi-format chirp waveforms are required. In [18], a photonic method employing a dual polarization dual-parallel Mach-Zehnder modulator (DP-DPMZM) was 
proposed to generate switchable dual-, down-, and up-chirp RF waveforms. In this method, signal switching was achieved by adjusting the phase of the input RF signal and chirp signal. In [19], a novel photonic approach for generating multi-format chirp waveforms based on a DP-MZM was demonstrated, in which switching among the dual- down-and up-chirp waveforms could be easily realized by adjusting the bias voltage on the DP-MZM. However, the generated multi-format chirp waveforms in the above approaches suffer from dispersion-induced RF power fading. In some situations where the generated signals are distributed to remote antenna sites based on radio over fiber [20], the capability of anti-dispersion transmission is highly demanded.

In this paper, we propose a novel photonic scheme to generate switchable multiformat chirp waveforms based on a dual-drive dual-parallel Mach-Zehnder modulator (DD-DPMZM). The generated signals are immune to dispersion-induced power fading. A full analytical model is presented to characterize the approach. An experiment was implemented to show the generation of multi-format chirp waveforms and switching among the dual-, down-, and up-chirp waveforms. An additional experiment on dispersive transmission along a $120 \mathrm{~km}$ optical fiber was also implemented, which verified the immunity of the dispersion-induced RF power fading. The major advantage of the approach lies in its relatively simple architecture, despite its powerful capability for signal generation, switching and anti-dispersion transmission. There is no polarization control and optical filtering in the given scheme, which also improves the stability and feasibility of the approach.

\section{Operation Principle and Theoretical Analysis}

The schematic diagram of the proposed scheme is shown in Figure 1. The system mainly consists of a continuous-wave (CW) laser source, a DD-DPMZM, a photodetector (PD), and an electrical bandpass filter (EBPF). In the DD-DPMZM, the lightwave from the laser source is split into two branches via a built-in $-3 \mathrm{~dB}$ coupler, and there is a dual-drive Mach-Zehnder modulator (DDMZM) in each branch. An RF signal $V_{1} \cos \left(\omega_{1} t\right)$ are injected into the two ports of DDMZM1 with a 90-degree phase difference by using a 90degree hybrid coupler, where $V_{1}$ and $\omega_{1}$ are the amplitude and frequency of the RF signal, respectively. In the DDMZM2, a single-chirped signal $V_{2} \cos \left(\omega_{2} t+k t^{2}\right)$ is injected into the two ports with a 90-degree phase difference, where $V_{2}, \omega_{2}$ and $k$ are the amplitude, center angular frequency and chirp rate of the single-chirped signal, respectively. We use $V_{\mathrm{B} 1}$, $V_{\mathrm{B} 2}$ and $V_{\mathrm{B} 3}$ to denote the bias voltages on DDMZM1, DDMZM2 and the main modulator, respectively. After the transmission along dispersive optical fiber, the optical signal is detected by the PD. The EBPF is used to filter out the unwanted harmonic components.

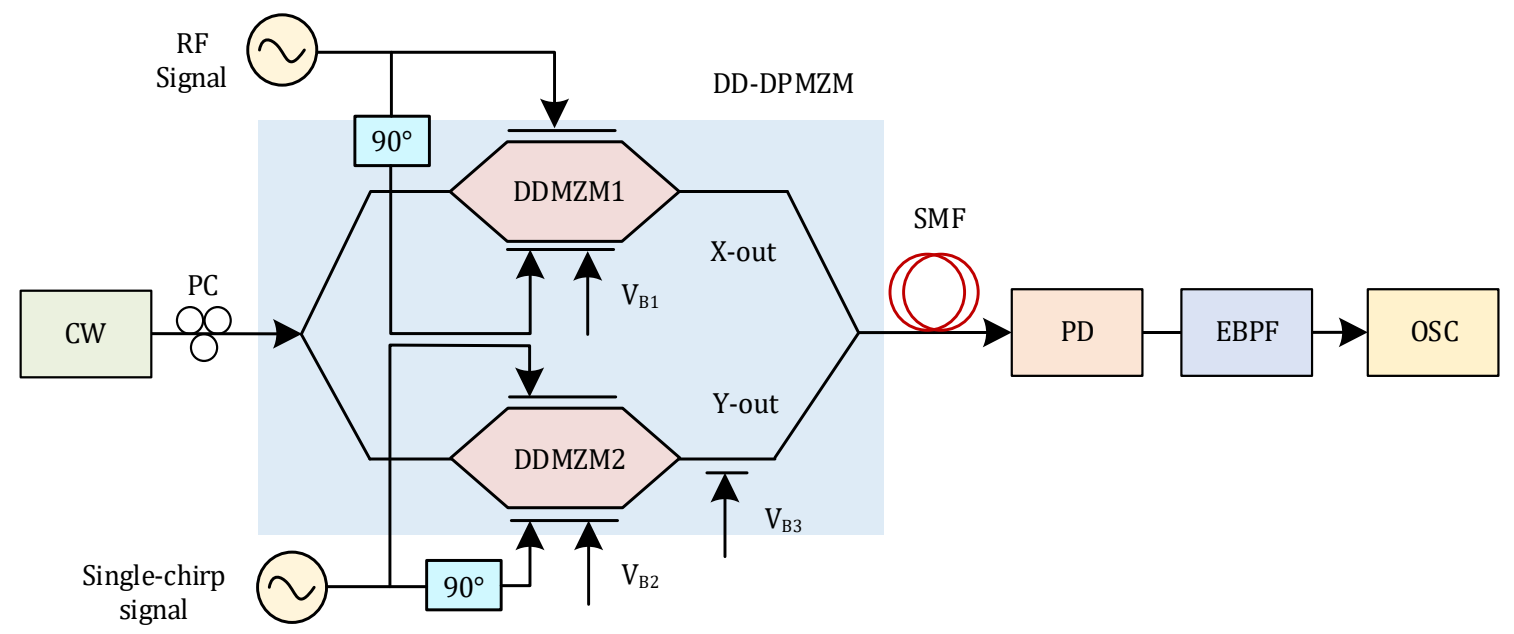

Figure 1. Schematic diagram of the proposed scheme. CW, continuous-wave source; PC, polarization controller; DD-DPMZM, dual drive dual-parallel Mach-Zehnder modulator; PD, photodetector; EBPF, electrical bandpass filter; OSC, oscilloscope. 
The electrical field of the signal at the output of DDMZM1 can be expressed as:

$$
\begin{aligned}
E_{x-\text { out }} & =\frac{\sqrt{2} E_{0}}{4} e^{j \omega_{0} t}\left\{J_{1}\left(m_{1}\right) e^{j\left(-\omega_{1} t+\frac{\pi}{2}\right)}+J_{0}\left(m_{1}\right)+J_{1}\left(m_{1}\right) e^{j\left(\omega_{1} t+\frac{\pi}{2}\right)}\right. \\
& \left.+e^{j \varphi_{1}} \cdot\left[J_{1}\left(m_{1}\right) e^{j\left(-\omega_{1} t\right)}+J_{0}\left(m_{1}\right)+J_{1}\left(m_{1}\right) e^{j\left(\omega_{1} t+\pi\right)}\right]\right\}
\end{aligned}
$$

where $E_{0}$ and $\omega_{0}$ are the amplitudes and angular frequencies of the optical carrier, respectively; $V_{\pi}$ is the half-wave voltage of DDMZM; $m_{1}=\pi V_{1} / V_{\pi}$ is the RF signal modulation index; $\varphi_{1}$ is the phase shift introduced by $V_{\mathrm{B} 1}$; and $J_{n}(\cdot)$ is the $n$-order first-kind Bessel function. In the above equation, we have applied the Jacobi-Anger expansion and assumed small signal modulation.

Similarly, we set $V_{\mathrm{B} 2}$ at the quadrature transmission point (QTP), corresponding to a phase shift of $\frac{\pi}{2}$; the optical signal at the output of the DDMZM2 can be expressed as:

$$
E_{\mathrm{y}-\text { out }}=\frac{\sqrt{2} E_{0}}{4} e^{j \omega_{0} t} \cdot\left[J_{0}\left(m_{2}\right) e^{j \frac{\pi}{2}}+J_{0}\left(m_{2}\right)+2 J_{1}\left(m_{2}\right) e^{j\left(-\omega_{2}-k t^{2}+\frac{\pi}{2}\right)}\right]
$$

Therefore, at the output of the DD-DPMZM, the optical signal is:

$$
\begin{gathered}
E_{\text {all-out }}=\frac{E_{0}}{4} e^{j \omega_{0} t}\left\{J_{1}\left(m_{1}\right) e^{j\left(-\omega_{1} t+\frac{\pi}{2}\right)}+J_{0}\left(m_{1}\right)+J_{1}\left(m_{1}\right) e^{j\left(\omega_{1} t+\frac{\pi}{2}\right)}\right. \\
\left.+e^{j \varphi_{1}} \cdot\left[J_{1}\left(m_{1}\right) e^{j\left(-\omega_{1} t\right)}+J_{0}\left(m_{1}\right)+J_{1}\left(m_{1}\right) e^{j\left(\omega_{1} t+\pi\right)}\right]\right\} \\
+\frac{E_{0}}{4} e^{j \omega_{0} t} \cdot e^{j \varphi_{3}}\left[J_{0}\left(m_{2}\right) e^{j \frac{\pi}{2}}+J_{0}\left(m_{2}\right)+2 J_{1}\left(m_{2}\right) e^{j\left(-\omega_{2}-k t^{2}+\frac{\pi}{2}\right)}\right]
\end{gathered}
$$

where $m_{2}=\pi V_{2} / V_{\pi}$ is the chirp signal modulation index; $\varphi_{3}$ is the phase shift induced by $V_{\mathrm{B} 3}$. If $m_{1}=m_{2}, \omega_{1}=2 \omega_{2}$, Equation (3) can be simplified as:

$$
\begin{aligned}
E_{\text {all }- \text { out }}= & \frac{E_{0}}{4} e^{j \omega_{0} t}\left[2 J_{1}\left(m_{1}\right) \cos \left(\frac{\varphi_{1}-\frac{\pi}{2}}{2}\right) e^{j\left(-2 \omega_{2} t\right)} e^{j\left(\frac{\varphi_{1}+\frac{\pi}{2}}{2}\right)}+J_{0}\left(m_{1}\right)\left(\sqrt{2} e^{j\left(\frac{\pi}{4}+\varphi_{3}\right)}+2 \cos \left(\frac{\varphi_{1}}{2}\right) e^{j \frac{\varphi_{1}}{2}}\right)\right. \\
& \left.+2 J_{1}\left(m_{1}\right) e^{j\left(-\omega_{2} t-k t^{2}\right)} e^{j\left(\frac{\pi}{2}+\varphi_{3}\right)}+2 J_{1}\left(m_{1}\right) \cos \left(\frac{\varphi_{1}+\frac{\pi}{2}}{2}\right) e^{j\left(2 \omega_{2} t\right)} e^{j \pi} e^{j\left(\frac{\varphi_{1}-\frac{\pi}{2}}{2}\right)}\right]
\end{aligned}
$$

After the dispersive transmission, the optical signal can be given by:

$$
\begin{aligned}
E_{\text {all-out }}=\frac{E_{0}}{4} e^{j \omega_{0} t}\left[2 J_{1}\left(m_{1}\right)\right. & \cos \left(\frac{\varphi_{1}-\frac{\pi}{2}}{2}\right) e^{j\left(-2 \omega_{2} t\right)} e^{j\left(\frac{\varphi_{1}+\frac{\pi}{2}}{2}+\gamma_{-1}\right)}+2 J_{1}\left(m_{1}\right) e^{j\left(-\omega_{2} t-k t^{2}\right)} e^{j\left(\frac{\pi}{2}+\varphi_{3}+\theta_{-1}\right)} \\
& +J_{0}\left(m_{1}\right)\left(\sqrt{2} e^{j\left(\frac{\pi}{4}+\varphi_{3}\right)}+2 \cos \left(\frac{\varphi_{1}}{2}\right) e^{j \frac{\varphi_{1}}{2}}\right) e^{j \theta_{0}} \\
+ & \left.2 J_{1}\left(m_{1}\right) \cos \left(\frac{\varphi_{1}+\frac{\pi}{2}}{2}\right) e^{j\left(2 \omega_{2} t\right)} e^{j\left(\frac{\varphi_{1}-\frac{\pi}{2}}{2}+\gamma_{1}+\pi\right)}\right]
\end{aligned}
$$

where $\theta_{0}$ and $\theta_{-1}$ are the chromatic-dispersion-induced phase shifts of the optical carrier and the first-order chirp signal optical sideband, respectively; $\gamma_{ \pm 1}$ is the dispersion-induced phase shifts of the first and first-order RF signal optical sidebands. Assuming the propagation constant of the dispersive fiber is given by $\beta$, the dispersion-induced phase shifts can be given by $\theta_{0}=L \beta_{0}\left(\omega_{0}\right), \theta_{-1}=L \beta_{0}\left(\omega_{0}\right)+L \beta_{1}\left(\omega_{0}\right)\left(-\omega_{2}\right)+\frac{1}{2} L \beta_{2}\left(\omega_{0}\right)\left(-\omega_{2}\right)^{2}$, and $\gamma_{ \pm 1}=L \beta_{0}\left(\omega_{0}\right) \pm L \beta_{1}\left(\omega_{0}\right)\left(2 \omega_{2}\right)+\frac{1}{2} L \beta_{2}\left(\omega_{0}\right)\left(2 \omega_{2}\right)^{2}$, where $L$ is the fiber length. After photodetection, by using a suitable EBPF, we can filter the signal to only include the spectral component centered at $\omega_{2}$. The filtered signal can be expressed as:

$$
\begin{aligned}
i(t) \propto & J_{0}\left(m_{1}\right) J_{1}\left(m_{1}\right)\left[\sqrt{2} \cos \left(\omega_{2} t+k t^{2}+\theta_{0}-\theta_{-1}+a_{1}-a_{2}\right)\right. \\
& \left.+2 \cos \left(\frac{\varphi_{1}}{2}\right) \cos \left(\omega_{2} t+k t^{2}+\theta_{0}-\theta_{-1}+a_{1}+a_{2}\right)\right] \\
+ & J_{1}\left(m_{1}\right)^{2} \cos \left(\frac{\varphi_{1}-\frac{\pi}{2}}{2}\right) \cos \left(\omega_{2} t-k t^{2}+\theta_{-1}-\gamma_{-1}+a_{3}\right)
\end{aligned}
$$

where $a_{1}=\frac{\varphi_{1}}{4}-\frac{3 \pi}{8}-\frac{\varphi_{3}}{2}, a_{2}=\frac{\varphi_{1}}{4}-\frac{\pi}{8}-\frac{\varphi_{3}}{2}, a_{3}=\varphi_{3}+\frac{\pi}{4}-\frac{\varphi_{1}}{2}$.

When we set the bias phase shifts as $\varphi_{1}=\frac{\pi}{2}$ and $\varphi_{3}=2 \cos ^{-1} \frac{J_{1}\left(m_{1}\right)}{\sqrt{2} J_{0}\left(m_{1}\right)}$, the output electrical signal can be expressed as:

$$
\begin{aligned}
& i(t) \propto J_{1}\left(m_{1}\right)^{2} \cos \left(\omega_{2} t+k t^{2}+a_{1}+\theta_{0}-\theta_{-1}\right) \\
& +J_{1}\left(m_{1}\right)^{2} \cos \left(\omega_{2} t-k t^{2}+a_{3}+\theta_{-1}-\gamma_{-1}\right)
\end{aligned}
$$


This is a dual-chirp signal. When $\varphi_{1}=\frac{\pi}{2}$ and $\varphi_{3}=\pi$, the signal is:

$$
i(t) \propto J_{1}\left(m_{1}\right)^{2} \cos \left(\omega_{2} t-k t^{2}+\pi+\theta_{-1}-\gamma_{-1}\right)
$$

This is a down-chirp signal. When $\varphi_{1}=-\frac{\pi}{2}$ and $\varphi_{3}=-\frac{\pi}{2}$, the signal is:

$$
i(t) \propto J_{1}\left(m_{1}\right) J_{0}\left(m_{1}\right) \cos \left(\omega_{2} t+k t^{2}-\frac{\pi}{4}+\theta_{0}-\theta_{-1}\right)
$$

This is an up-chirp signal. Therefore, we can generate the dual-, down, and up-chirp signals by properly setting $V_{\mathrm{B} 1}$ and $V_{\mathrm{B} 2}$. The process of the generation of multi-format chirp signals is shown in Figure 2. It can be seen that the generated signals are all single-sidebandmodulated, and are immune to the dispersion-induced RF power fading. The dispersion in fiber only affects the initial phases of the generated up-, down- and dual-chirp signals, rather than their amplitude. In other words, the generated up-, down- and dual-chirp signals are capable of anti-dispersion transmission.

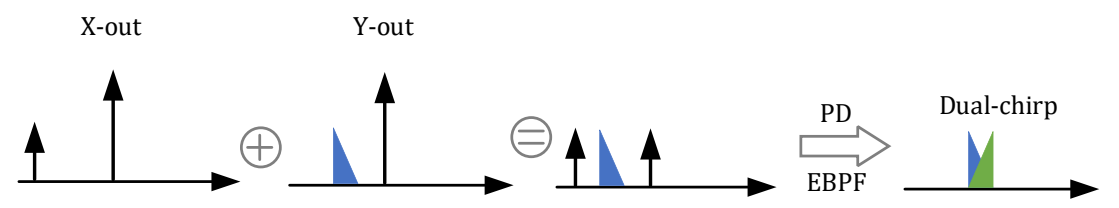

(a)

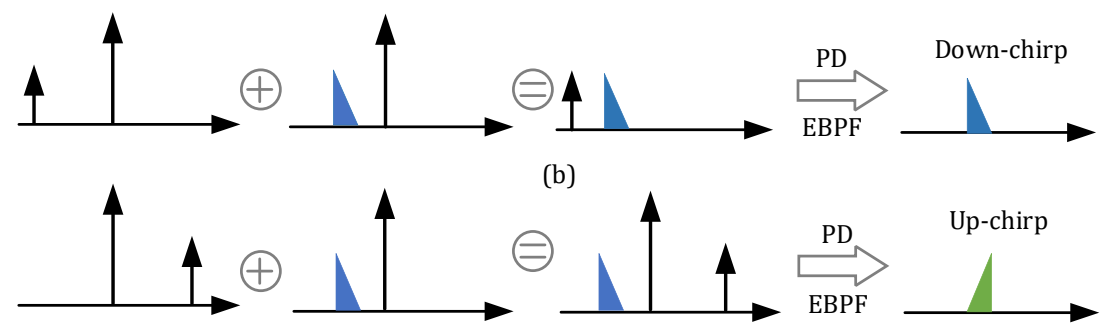

(c)

Figure 2. Spectral illustration of the generation of multi-format chirp signals. (a) Dual-chirp signal. (b) Down-chirp signal. (c) Up-chirp signal.

\section{Experimental Results}

A proof-of-concept experiment with the setup shown in Figure 1 was implemented. A tunable laser source (ID Photonics, CoBrite-DX) emitted a CW light with a linewidth of $25 \mathrm{kHz}$ at a wavelength of $1550.1 \mathrm{~nm}$, which was injected into a DD-DPMZM (FTM7960EX/ 301). A polarization controller was placed in front of the modulator to minimize the polarization-dependent loss. Utilizing a 90-degree hybrid coupler, a $5 \mathrm{GHz}$ RF signal from a signal generator (R\&S, SMB 100A) was applied to the two ports of the DDMZM1 within the DD-DPMZM. A chirp signal with a time duration of $1 \mu \mathrm{s}$, a bandwidth of $0.5 \mathrm{GHz}$, and a center frequency of $2.5 \mathrm{GHz}$, from an arbitrary waveform generator (Keysight, M8190A) was applied to the DDMZM2, also with a 90-degree phase difference between the two ports. An avalanche photodiode (APD) with a $3 \mathrm{~dB}$ bandwidth of $10 \mathrm{GHz}$ was employed to perform O/E conversion. The detected signal was then recorded with a $20 \mathrm{GS} / \mathrm{s}$ real-time oscilloscope (R\&S, RTP084). Both the bandpass filtering and the time-frequency analyses were performed with an offline computer program.

At the beginning of the experiment, we verified the generation of the dual-, down- and up-chirp signals. We set the amplitude of the input unmodulated signal and chirp signal to both be $0.8 \mathrm{~V}$ in order to satisfy $m_{1}=m_{2}$, and set $V_{\mathrm{B} 2}$ to be $2.4 \mathrm{~V}$, corresponding to the quadrature transmission point of DDMZM2. First, we set Bias1 to be $2.4 \mathrm{~V}$, corresponding to a phase shift of $\varphi_{1}=\frac{\pi}{2}$, and set $V_{\mathrm{B} 3}$ to be $5.2 \mathrm{~V}$, corresponding to $\varphi_{3}=2 \cos ^{-1} \frac{J_{1}\left(m_{1}\right)}{\sqrt{2} J_{0}\left(m_{1}\right)}$. The observed time-domain waveform, time-frequency diagram obtained by the short- 
time Fourier-transform and the spectrum of the generated signal are given in Figure 3a-c, respectively. It is seen that a dual-chirp signal, with a center frequency of $2.5 \mathrm{GHz}$, a bandwidth of $0.5 \mathrm{GHz}$, and a duration of $1 \mu \mathrm{s}$, has been generated successfully. Next, $V_{\mathrm{B} 1}$ remains unchanged, and we adjusted $V_{\mathrm{B} 3}$ to be $6.5 \mathrm{~V}$, corresponding to $\varphi_{3}=\pi$. The observed time-domain waveform, time-frequency diagram and spectrum of the generated signal are given in Figure $3 \mathrm{~d}-\mathrm{f}$, respectively. It can be seen that a high-quality down-chirp signal was generated. Finally, we set $V_{\mathrm{B} 1}$ to be $10.9 \mathrm{~V}$, corresponding to $\varphi_{1}=-\frac{\pi}{2}$, and set $V_{\mathrm{B} 3}$ to be $10.7 \mathrm{~V}$, corresponding to $\varphi_{3}=-\frac{\pi}{2}$. The results are given in Figure $3 \mathrm{~g}-\mathrm{i}$. Thus far, we have generated high-quality dual-, down- and up-chirp signals with a center frequency of $2.5 \mathrm{GHz}$, a bandwidth of $0.5 \mathrm{GHz}$, and a duration of $1 \mu$ s by properly setting the bias voltages on the modulator.

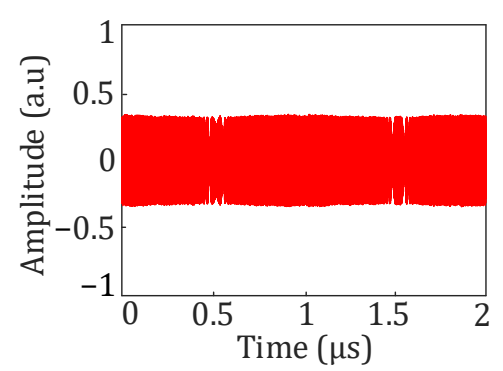

(a)

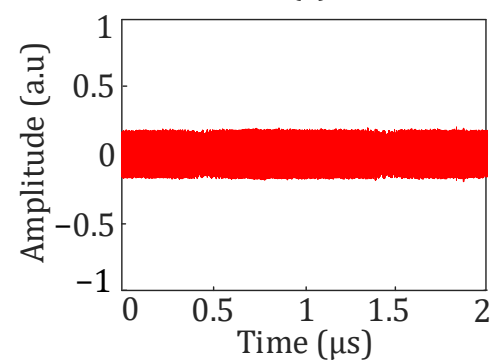

(d)

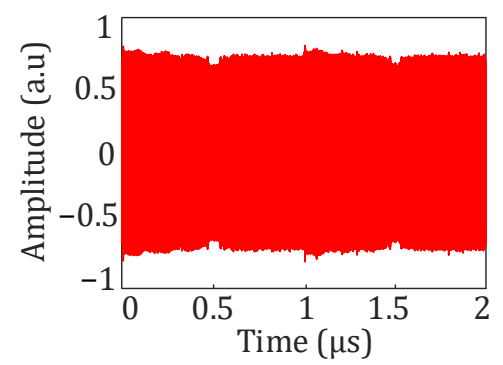

(g)

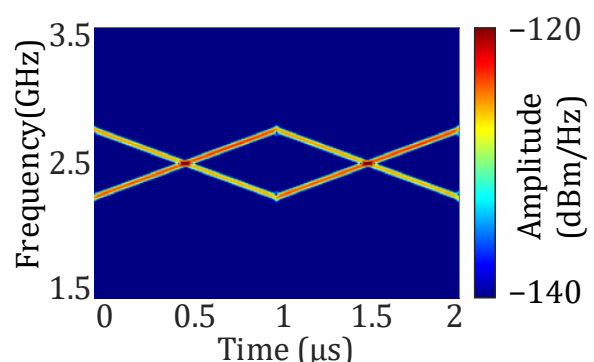

(b)

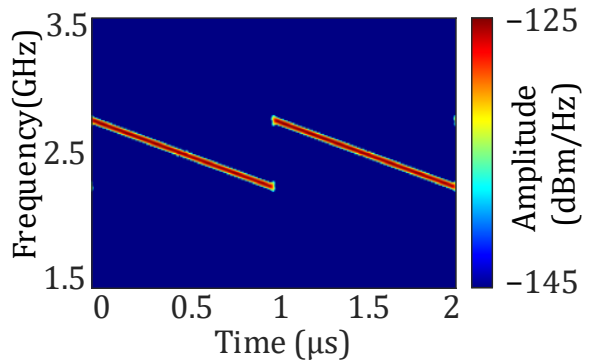

(e)

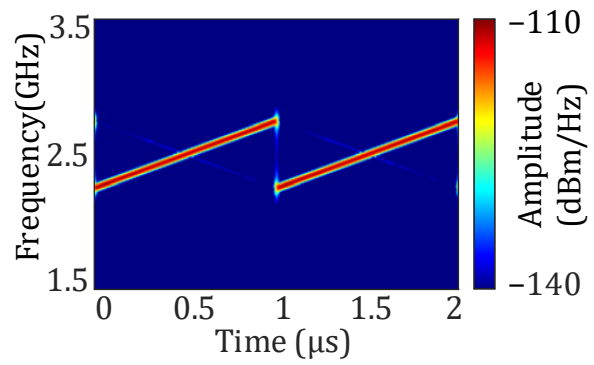

(h)

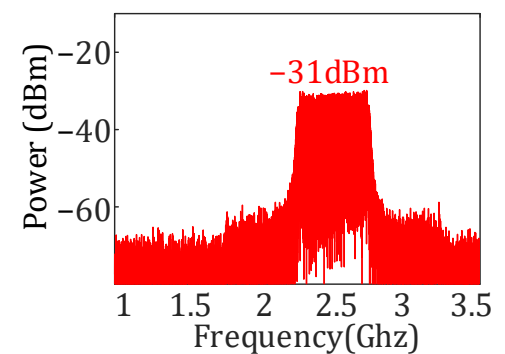

(c)

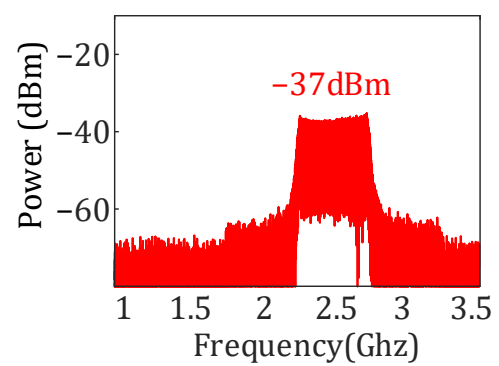

(f)

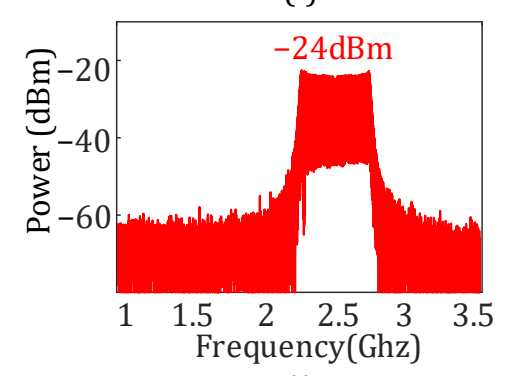

(i)

Figure 3. (a,d,g) Time-domain waveforms of the generated dual-, down- and up-chirp signals; (b,e,h) time-frequency diagrams; (c,f,i) spectra.

Next, in order to test the anti-dispersion transmission of the given approach, a dispersion compensating module (DCM) with a dispersion equivalent to a $120 \mathrm{~km}$ standard single-mode fiber was placed after the DD-DPMZM. The insertion loss of the DCM was around $9 \mathrm{~dB}$. The observed time-frequency diagrams and spectra of the generated multiformat chirp signals are shown in Figure 4. Again, it is seen that high-quality dual-, downand up-chirp signals were obtained. The powers of the generated the dual-, down- and up-chirp signals were approximately $-47 \mathrm{dBm},-53 \mathrm{dBm}$ and $-40 \mathrm{dBm}$, respectively. Compared to the former case without long fiber transmission, there was a $16 \mathrm{~dB}$ power reduction, which was due to the insertion loss of the DCM, but not the dispersion effect. Then, the DCM was replaced by an optical attenuator with a $9 \mathrm{~dB}$ insertion loss. The results are shown in Figure 5. It can be observed that the time-frequency diagrams and the 
electrical spectra remained almost unchanged as compared to Figure 4. Especially, the RF powers were the same as the case with dispersive transmission. From these results, we can conclude that the scheme was immune to dispersion-induced RF power fading, which is highly desirable in distributed radar systems based on radio transmission over fibers.

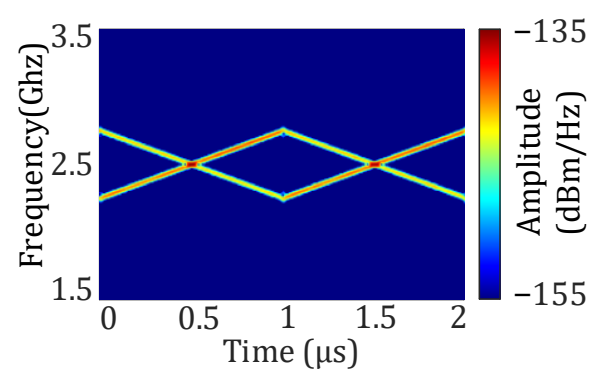

(a)

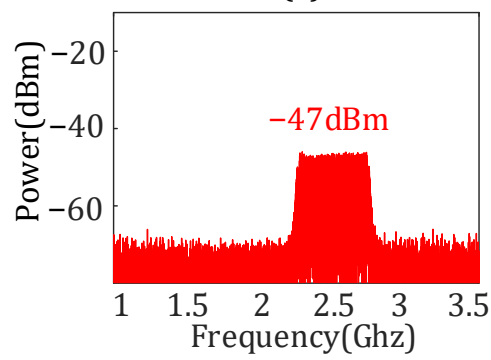

(d)

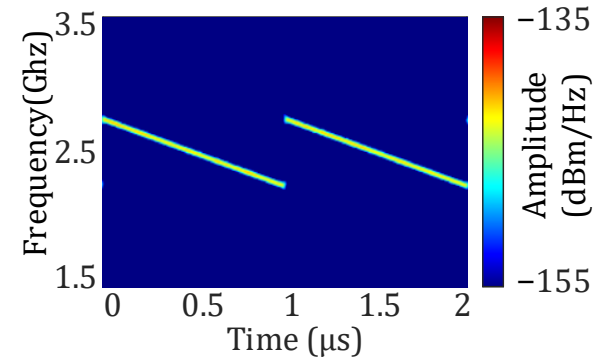

(b)

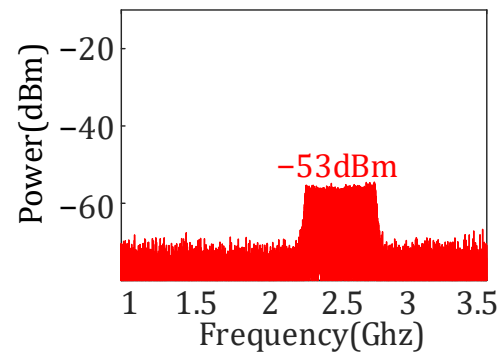

(e)

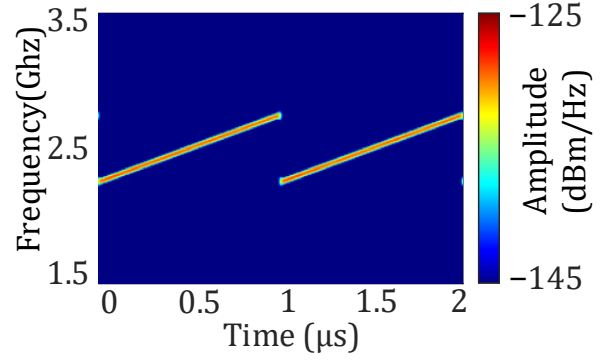

(c)

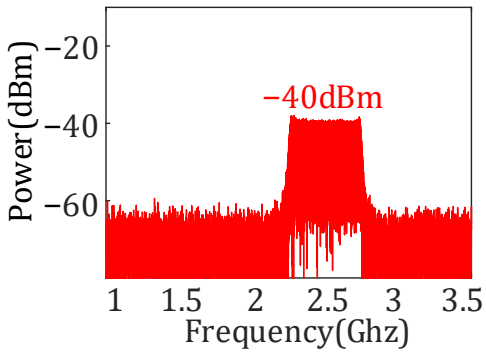

(f)

Figure 4. (a-c) Time-frequency diagrams of the generated dual-, down- and up-chirp signals after dispersive transmission; (d-f) spectra.

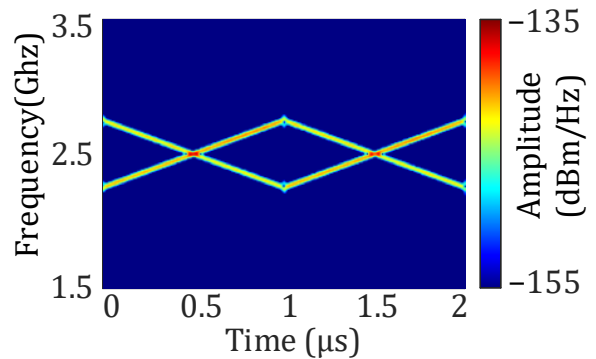

(a)

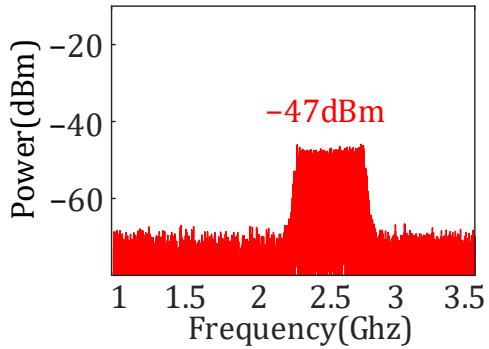

(d)

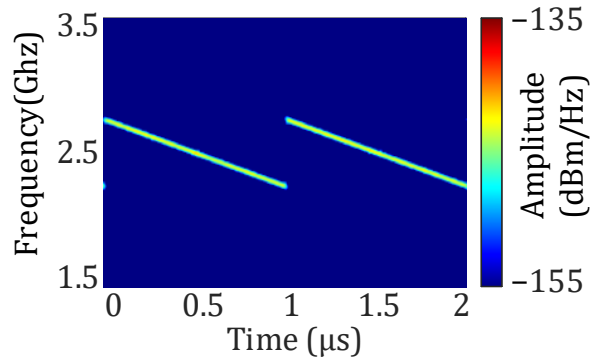

(b)

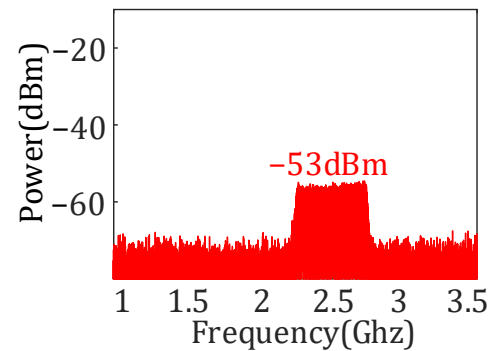

(e)

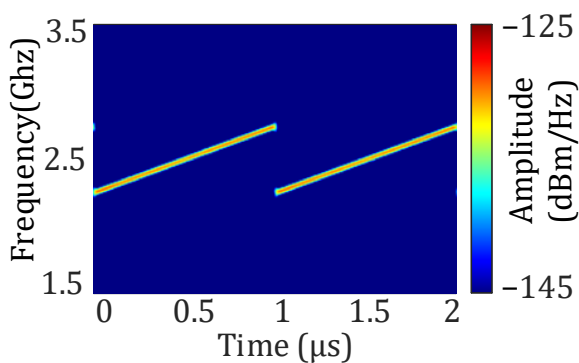

(c)

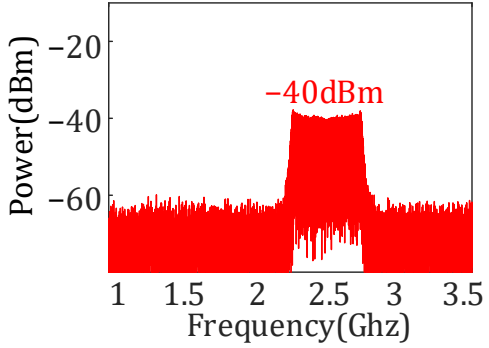

(f)

Figure 5. (a-c) Time-frequency diagrams of the generated dual-, down- and up-chirp signals after optical attenuation; (d-f) spectra.

\section{Discussion}

Compared with the methods proposed in [18], our scheme uses a DD-DPMZM, which simplifies the system structure. In [18], switching among the dual-, up- and down-chirp signals was accomplished by changing the phase shift of the phase shifter. However, in our scheme, we only need to change the bias voltages on the modulator, which is easier to implement. The scheme in [19] can complete the switching among the dual-, up- and 
down-chirp signals by adjusting the bias voltages on a DP-MZM, but the generated upchirp and down-chirp signals in [19] had large differences in amplitude. In our scheme, the up- and down-chirp signals in the dual-chirp had almost the same amplitude, which can be observed in Figure 3b. Most importantly, in references [18,19], the generated multi-format chirp waveforms suffered from dispersion-induced RF power fading. In contrast, our scheme featured anti-dispersion transmission.

In our experiment, the drift of the bias voltages cannot be ignored. When $V_{\mathrm{B} 1}$ and $V_{\mathrm{B} 2} \mathrm{drift}$, it will affect the performance of single-sideband modulation, which weakens the anti-dispersion function of the switchable multi-format chirp signal. On the other hand, the drift of $V_{\mathrm{B} 3}$ will affect the power of the generated up-chirp signal and the power difference between the up- and down-chirp signals in the dual-chirp signal. It also brings some unwanted spectral components into the generated down-chirp signal. The waveforms generated in our experiment could remain almost unchanged for more than ten minutes. We did not use an automatic bias controller in the experiment. If long-term stability is required in practice, an automatic bias controller is necessary.

\section{Conclusions}

In summary, a novel photonic approach to generating the switchable multi-format chirp waveforms based on a DD-DPMZM has been proposed. A major advantage is its immunity to dispersion-induced RF power fading. By properly controlling the bias voltages on the DD-DPMZM, different chirp RF waveforms, including dual-, up- and down-chirp waveforms, can be obtained. We have provided full theoretical analysis on the principle of the scheme. The generation and switching of multi-format chirp waveforms, as well as their anti-dispersion transmission, have been verified in an experimental demonstration. Our method has relatively simple architecture and there is no polarization control and optical filtering. We believe the proposed scheme to be a potential solution to the generation of switchable multi-format chirp waveforms in radio-over-fiber-based distributed radar systems.

Author Contributions: Conceptualization, J.O. and H.C.; investigation, C.H.; data curation, C.H. and H.C.; writing-original draft preparation, C.H. and H.C.; writing—review and editing, C.H. and H.C. All authors have read and agreed to the published version of the manuscript.

Funding: This work was supported by the National Natural Science Foundation of China (NSFC) (61975048, 41905024), Natural Science Foundation of Zhejiang Province (LZ20F010003, LQ19F010008).

Institutional Review Board Statement: Not applicable.

Informed Consent Statement: Not applicable.

Data Availability Statement: The data presented in this study are available on request from the corresponding author. The data are not publicly available due to the data also forming part of an ongoing study.

Acknowledgments: The authors express their appreciation to the anonymous reviewers for their valuable suggestions.

Conflicts of Interest: The authors declare no conflict of interest.

\section{References}

1. Richards, M.A.; Scheer, J.; Holm, W.A.; Melvin, W.L. Principles of Modern Radar; Scitech Publising, Inc.: Realeigh, NC, USA, 2010.

2. Skolnik, M. Role of radar in microwaves. IEEE Trans. Microw. Theory 2002, 50, 625-632. [CrossRef]

3. Zhu, D.; Pan, S. Broadband cognitive radio enabled by photonics. J. Lightwave Technol. 2020, 38, 3076-3088. [CrossRef]

4. Chi, H.; Wang, C.; Yao, J. Photonic generation of wideband chirped microwave waveforms. IEEE J. Microw. $2021,1,787-803$. [CrossRef]

5. Zeitouny, A.; Stepanov, S.; Levinson, O.; Horowitz, M. Optical generation of linearly chirped microwave pulses using fiber Bragg gratings. IEEE Photonic. Technol. Lett. 2005, 17, 660-662. [CrossRef]

6. Wang, C.; Yao, J. Photonic generation of chirped microwave pulses using superimposed chirped fiber Bragg gratings. IEEE Photonic. Technol. Lett. 2008, 20, 882-884. [CrossRef] 
7. Chi, C.; Yao, J. Chirped RF pulse generation based on optical spectral shaping and wavelength-to-time mapping using a nonlinearly chirped fiber Bragg grating. J. Lightwave Technol. 2008, 26, 1282-1287. [CrossRef]

8. Wang, C.; Yao, J. Large time-bandwidth product microwave arbitrary waveform generation using a spatially discrete chirped fiber Bragg grating. J. Lightwave Technol. 2010, 28, 1652-1660. [CrossRef]

9. Herrera, L.E.Y.; Ribeiro, R.M.; Jabulka, V.B.; Tovar, P.; von der Weid, J.P. Photonic generation and transmission of linearly chirped microwave pulses with high TBWP by self-heterodyne technique. J. Lightwave Technol. 2018, 36, 4408-4415. [CrossRef]

10. Zhang, Y.; Ye, X.; Guo, Q.; Zhang, F.; Pan, S. Photonic generation of linear-frequency-modulated waveforms with improved time-bandwidth product based on polarization modulation. J. Lightwave Technol. 2017, 35, 1821-1829. [CrossRef]

11. Kanno, A.; Kawanishi, T. Broadband frequency-modulated continuous-wave signal generation by optical modulation technique J. Lightwave Technol. 2014, 32, 3566-3572. [CrossRef]

12. Zhou, P.; Zhang, F.; Pan, S. Generation of linear frequency-modulated waveforms by a frequency-sweeping optoelectronic oscillator. J. Lightwave Technol. 2018, 36, 3927-3934. [CrossRef]

13. Zhang, H.; Wang, S.; Jia, S.; Yu, X.; Jin, X.; Zheng, S.; Chi, H.; Zhang, X. Experimental generation of linearly chirped $350 \mathrm{GHz}$ band pulses with a bandwidth beyond $60 \mathrm{GHz}$. Opt. Lett. 2017, 42, 5242-5245. [CrossRef] [PubMed]

14. Liu, Y.; Liang, J.; Li, X.; Xiao, N.; Zhang, Z.; Yuan, X. Theoretical investigation of photonic generation of frequency quadrupling linearly chirped waveform with large tunable range. Opt. Express 2017, 25, 16196-16203. [CrossRef] [PubMed]

15. Zhu, D.; Yao, J. Dual-chirp microwave waveform generation using a dual-parallel Mach-Zehnder modulator. IEEE Photonic. Technol. Lett. 2015, 27, 1410-1413. [CrossRef]

16. Xu, Y.; Jin, T.; Chi, H.; Zheng, S.; Jin, X.; Zhang, X. Photonic generation of dual-chirp waveforms with improved time-bandwidth product. IEEE Photonic. Technol. Lett. 2017, 29, 1253-1256. [CrossRef]

17. Li, X.; Zhao, S.; Zhu, Z.; Qu, K.; Lin, T.; Hu, D. Photonic generation of frequency and bandwidth multiplying dual-chirp microwave waveform. IEEE Photonics J. 2017, 9, 1-14. [CrossRef]

18. Li, P.; Yan, L.; Ye, J.; Zou, X.; Luo, B.; Pan, W. Photonic approach for the generation of switchable down-, up-, and dual-chirped linear frequency-modulated microwave signals. Opt. Lett. 2020, 45, 1990-1993. [CrossRef] [PubMed]

19. Yi, C.; Yang, S.; Yang, B.; Jin, T.; Chi, H. Photonic approach for generating bandwidth-doubled and switchable multi-format chirp waveforms. Opt. Lett. 2021, 46, 1578-1581. [CrossRef] [PubMed]

20. Malacarne, A.; Maresca, S.; Scotti, F.; Ghelfi, P.; Serafino, G.; Bogoni, A. Coherent dual-band radar-over-fiber network with VCSEL-based signal distribution. J. Lightwave Technol. 2020, 38, 6257-6264. [CrossRef] 